\title{
Advantages of the rapid double-staining method for intraoperative detection of micrometastasis in sentinel lymph nodes
}

\author{
TOSHIHIKO OJIMA ${ }^{1}$, SHINICHI KINAMI ${ }^{1}$, KEISHI NAKAMURA ${ }^{1}$, KATSUNOBU OYAMA $^{1}$, \\ MASASHI INOKUCHI $^{1}$, HIDETO FUJITA ${ }^{1}$, ITASU NINOMIYA ${ }^{1}$, SACHIO FUSHIDA ${ }^{1}$, \\ TAKASHI FUJIMURA ${ }^{1}$, SEIKO KITAMURA ${ }^{2}$, SHINICHI HARADA ${ }^{3}$ and TETSUO OHTA $^{1}$ \\ ${ }^{1}$ Gastroenterologic Surgery, Department of Oncology, ${ }^{2}$ Department of Pathology, and \\ ${ }^{3}$ Center for Biomedical Research and Education, Division of Cancer Medicine, Graduate School \\ of Medical Science, Kanazawa University, Kanazawa, Ishikawa 920-8641, Japan
}

Received April 18,2013; Accepted May 27, 2013

DOI: 10.3892/or.2013.2591

\begin{abstract}
For rapid intraoperative diagnosis of lymph node micrometastasis, we refined the rapid immunohistochemistry method by combining anti-cytokeratin antibody-labeled nanocrystal beads with rapid hematoxylin and eosin (H\&E) staining on the same section, referred to as the rapid double staining (RDS) technique. Two frozen-section slices each were obtained from 372 lymph nodes of 100 breast cancer patients. We performed RDS for 1 slide and rapid H\&E staining for the other. The results were compared with the corresponding final pathological data obtained from the permanent specimens. For specimens from patients with $\mathrm{pN} 1(\mathrm{mi})$ as determined by final pathological examination, the false-negative rate was $33.3 \%$ for rapid H\&E staining and $16.7 \%$ for RDS. For specimens from patients with $\mathrm{pNO}(\mathrm{i}+$ ) as determined by final pathological examination, the false-negative rate was $80 \%$ for rapid H\&E staining and $0 \%$ for RDS. These results indicate that RDS is superior to conventional rapid H\&E staining for intraoperative diagnosis of nodal micrometastasis and isolated tumor cells.
\end{abstract}

\section{Introduction}

The presence or absence of lymph node metastasis is an important prognostic factor in breast cancer (1). However, the significance of lymph node micro-involvement remains unclear $(2,3)$. In the UICC TNM classification (7th edition), micrometastases are defined as metastases with a long diameter of $2 \mathrm{~mm}$ or less as observed in section samples; those with diameters of $0.2 \mathrm{~mm}$ or less are defined as isolated tumor

Correspondence to: Dr Shinichi Kinami, Gastroenterologic Surgery, Department of Oncology, Division of Cancer Medicine, Graduate School of Medical Science, Kanazawa University, 13-1 Takaramachi, Kanazawa, Ishikawa 920-8641, Japan

E-mail:kinami@kanazawa-med.ac.jp

Key words: lymph node metastasis, sentinel node, nanocrystal beads, rapid immunohistochemistry cells (ITCs). While ITCs are not thought to affect outcomes, micrometastases are considered to be a prognostic factor (4).

In clinically node-negative breast cancer, the presence or absence of lymph node metastasis is primarily determined by sentinel node (SN) biopsy (5). When omitting axillary dissection based on SN biopsy results, detection of micrometastases using a rapid diagnostic method would be beneficial (4) However, the accuracy of conventional intraoperative diagnosis remains low. Although the false-negative rate could be reduced by preparing several 2 -mm-thick sections, the diagnostic potential of frozen sections is limited (6).

One of the current commonly used techniques for intraoperative diagnosis of nodal metastasis is rapid immunohistochemistry (IHC). Rapid IHC aims to improve the accuracy of frozen-section diagnoses. However, this method has drawbacks that make it difficult to use for the diagnosis of micrometastases and ITCs (7). Qualitative diagnosis of cells is poor given the lack of cytoplasmic staining, and sections used for rapid IHC are prepared differently from those used for hematoxylin and eosin (H\&E) staining (8).

Therefore, we refined the rapid IHC technique using nanocrystal beads labeled with an anti-cytokeratin antibody for determining lymph node metastasis (9). This method uses a double-staining procedure that combines rapid fluorescent immunostaining and H\&E staining on the same section. We refer to this method as 'RDS' (rapid double staining with H\&E and immuno-nanocrystal bead staining) (9). Using lymph nodes extracted from breast cancer patients who had undergone SN biopsies in our unit, we compared the false-negative rates of the RDS and rapid $\mathrm{H} \& \mathrm{E}$ methods.

\section{Materials and methods}

Nanocrystal beads and antibody. We used quantum dots (QDs) Qdot ${ }^{\circledR} 655$ (Invitrogen, Carlsbad, CA, USA) as nanocrystal beads (9). The cytokeratin 8 antibody SC-8020 (Santa Cruz Biotechnology, Santa Cruz, CA, USA) was adopted as the anti-cytokeratin antibody (8).

RDS procedure. QD labeling with anti-cytokeratin antibodies was carried out according to the specified protocol (Fig. 1) (10). 


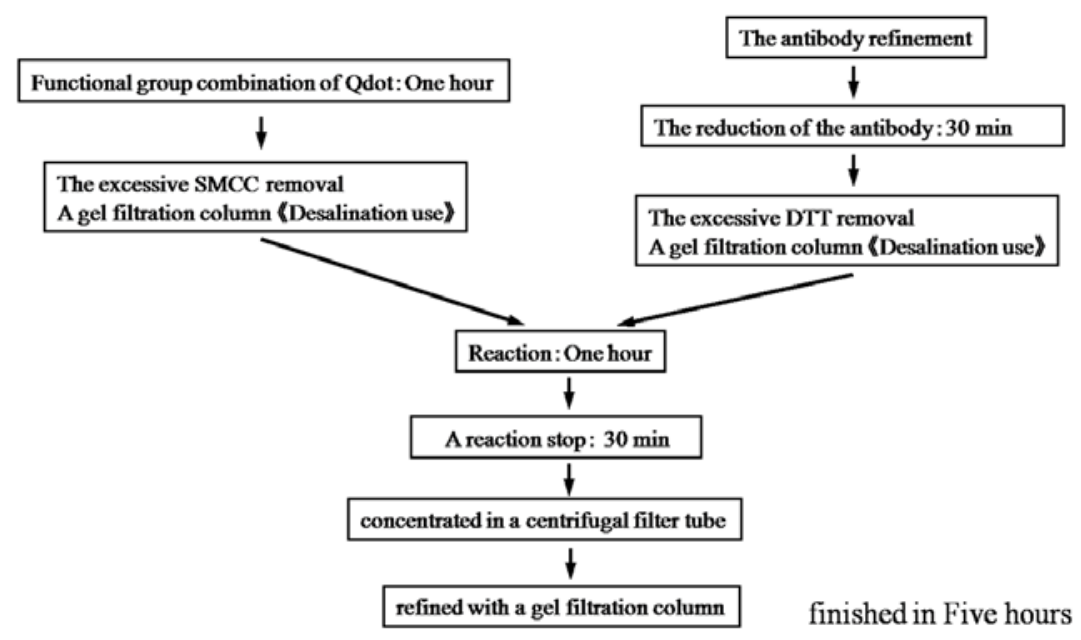

Figure 1. The $\operatorname{Qdot}^{\circledR}$ and antibody kit procedure.

Stock solutions of antibody-labeled QDs were stored at $4{ }^{\circ} \mathrm{C}$. The procedure for RDS was as follows. Frozen samples were first rapidly fixed for $30 \mathrm{sec}$ in $100 \%$ acetone, followed by rinsing with running water. After rinsing in phosphate-buffered saline (PBS) for $1 \mathrm{~min}$, a few drops of the 10-fold diluted QD solution were applied to the samples, which were then incubated at $37^{\circ} \mathrm{C}$ for $20 \mathrm{~min}$. After rinsing with PBS for $1 \mathrm{~min}$, samples were stained with hematoxylin for $10 \mathrm{sec}$. Samples were then rinsed with running water for $30 \mathrm{sec}$, followed by staining with eosin for $3 \mathrm{sec}$. Finally, the samples were fixed in alcohol for $1 \mathrm{~min}$ and sealed with xylene (Table I). The entire staining procedure can be completed within $30 \mathrm{~min}$ (8).

Observation of specimens. Fixed samples were examined under a fluorescence microscope BX51-3 (Olympus, Tokyo, Japan) equipped with a U-MWU2 optical filter (Olympus) (11). Both the bright-field views and the fluorescence views of the same microscopic field are able to be observed with the BX51-3. The observation method for the RDS slide was as follows. We first looked for any fluorescent sites with the fluorescent view. If any such site was found, we switched to bright-field observation and examined whether it was a metastatic focus. Both bright-field and fluorescent images are able to be observed merely by switching filters.

Patients and lymph nodes. Samples used in the present study were from 372 lymph nodes from 100 patients who suffered from breast cancer. Patients underwent partial mastectomy and SN biopsy without neoadjuvant chemotherapy between October 2007 and March 2009. Our institute adopts combination mapping with radioisotope (RI) and blue dye to identify $\mathrm{SN}$ in breast cancer patients (12). For the RI tracer, we perform subcutaneous injection around the tumor; for the dye, Patent Blue is subcutaneously injected beneath the areola.

For SNs dissected during surgery, multiple 2-mm slices were cut parallel to the central slice containing the hilus. Two sets of sections for pathological diagnosis during surgery, one for conventional rapid H\&E staining and the other for RDS, were prepared in the pathology department of our hospital. We compared the false-negative rates of the rapid H\&E and RDS methods using the final pathology results from permanent
Table I. Procedure for the hematoxylin and eosin and immunonanocrystal bead staining.

\begin{tabular}{rl}
\hline Step & \multicolumn{1}{c}{ Procedure } \\
\hline 1 & Acetone solution for 1 min \\
2 & Rinse with water for 30 sec \\
3 & Rinse with PBS one time \\
4 & Staining with labeled Qdot diluted 20 times \\
& for 20 min at $37^{\circ} \mathrm{C}$ \\
5 & Rinse with PBS twice \\
6 & Hematoxylin for $10 \mathrm{sec}$ \\
7 & Rinse with water for 30 sec \\
8 & Eosin for 3 sec \\
9 & Rinse with water for 30 sec \\
10 & $99 \%$ alcohol for $1 \mathrm{~min}$ \\
11 & Enclose in xylene \\
\hline
\end{tabular}

preparations as a reference. The final pathology results were categorized as metastasis (pN1), micrometastasis [pN1(mi)], or isolated tumor cells [pNO(i+)], according to the UICC classification (7th edition). In our pathology department, the diagnostic procedure for final pathology results involves diagnosing metastases using H\&E-stained, formalin-fixed permanent preparations; for lymph nodes diagnosed as n0, including ITCs, cytokeratin immunostaining is then carried out to confirm negative diagnoses of metastasis (12).

Ethical guidelines of the study. All the patients were fully informed in regards to the purpose and content of the study and provided informed consent for participation in the study, according to the Helsinki Declaration.

\section{Results}

Detection of metastasis by RDS. Fig. 2 shows the images of metastatic lymph nodes stained by RDS. In the bright-field observation, metastatic foci of lymph nodes were observed 
A

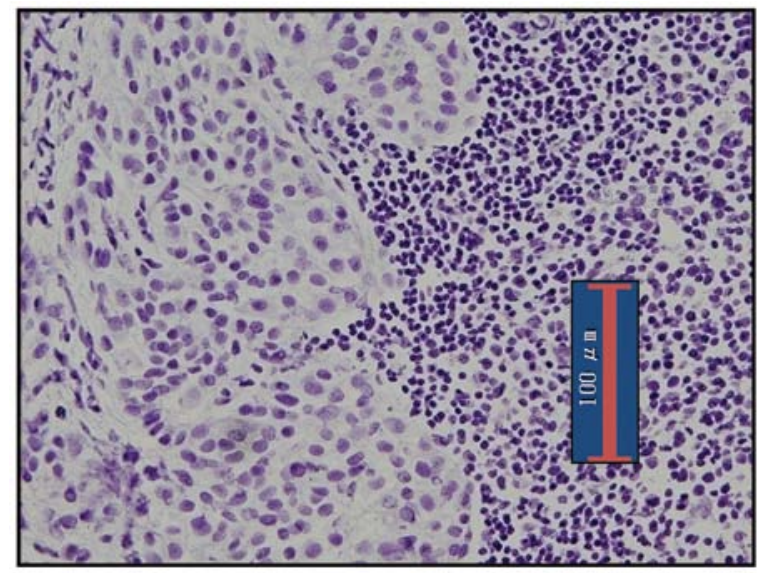

B

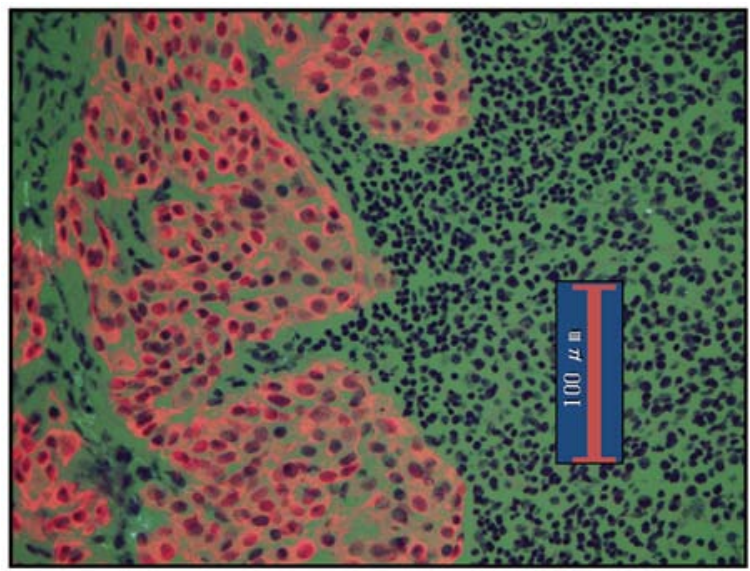

Figure 2. Images of metastatic lymph nodes in the breast cancer cases. (A) Transmission bright-field observation; (B) epifluorescence.

A

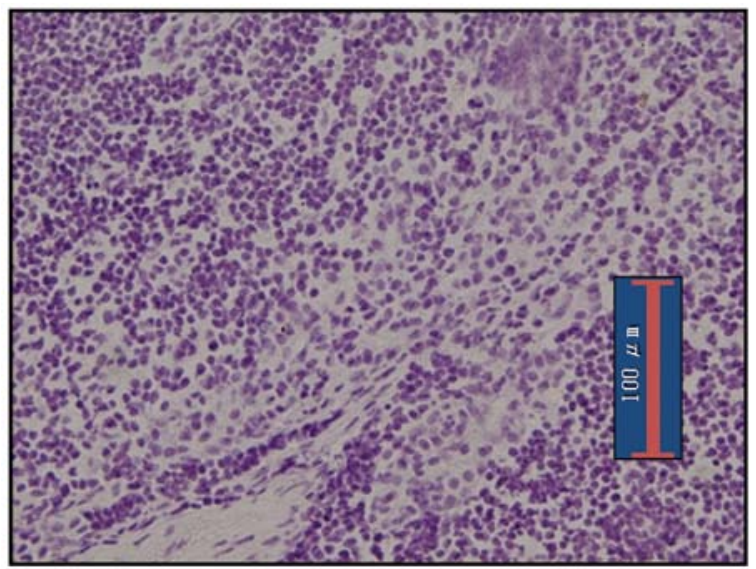

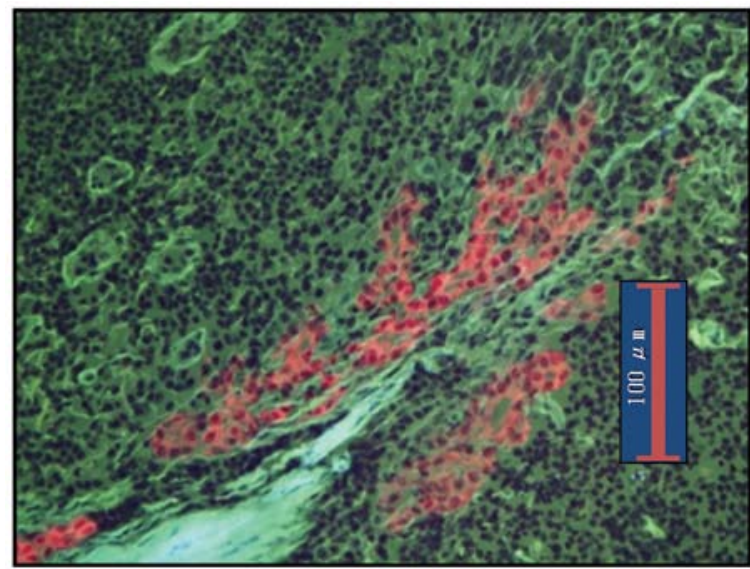

Figure 3. Images of micrometastatic lymph nodes in the breast cancer cases. (A) Transmission bright-field observation; (B) epifluorescence.

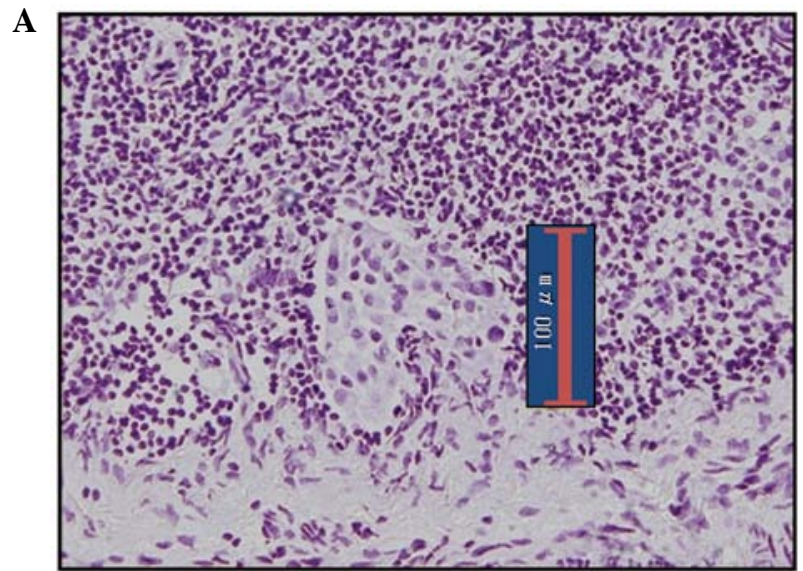

B

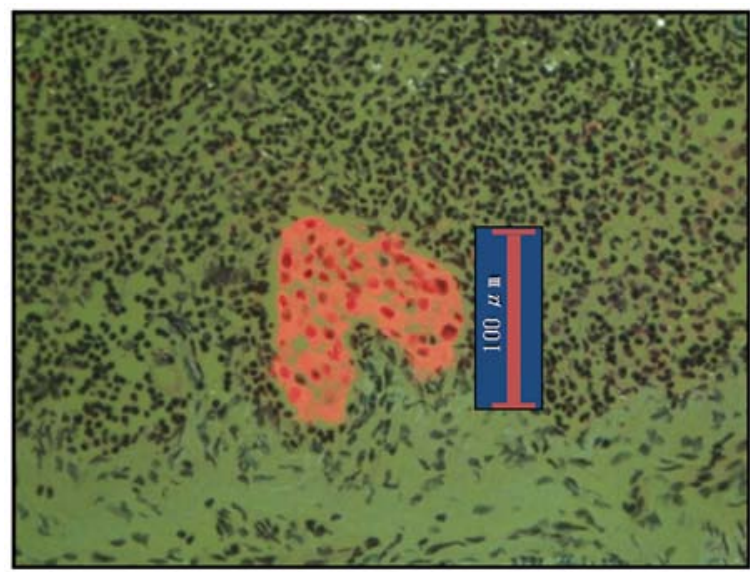

Figure 4. Images of isolated tumor cells in the breast cancer cases. (A) Transmission bright-field observation; (B) epifluorescence.

with H\&E staining. For observation of fluorescence, the cytoplasm of cancer cells was clearly stained orange by the QDs, while normal lymphocytes appeared green due to eosin staining. This provides a clear contrast that facilitates the diagnosis of metastasis.

RDS images of micrometastasis in lymph nodes are shown in Fig. 3. As in pN1(mi) nodes, making a diagnosis of metastasis is easy. Fig. 4 shows ITC images detected by RDS. The diagnosis of ITCs are known to be somewhat difficult by bright-field observation; however, in our series ITCs were readily diagnosable with observation of epifluorescence given the clear contrast between cancerous and normal cells.

Fig. 5 shows plasma cells in lymph nodes. Since anticytokeratin antibodies react with plasma cells, they sometimes 
A

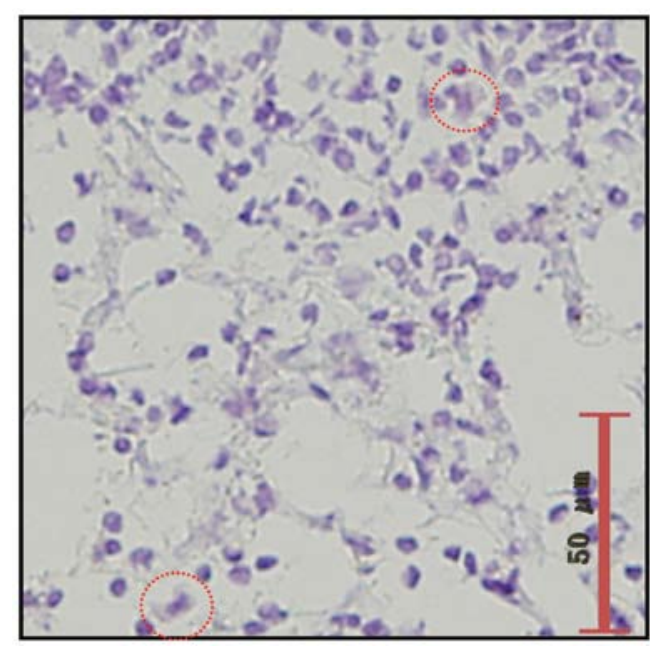

B

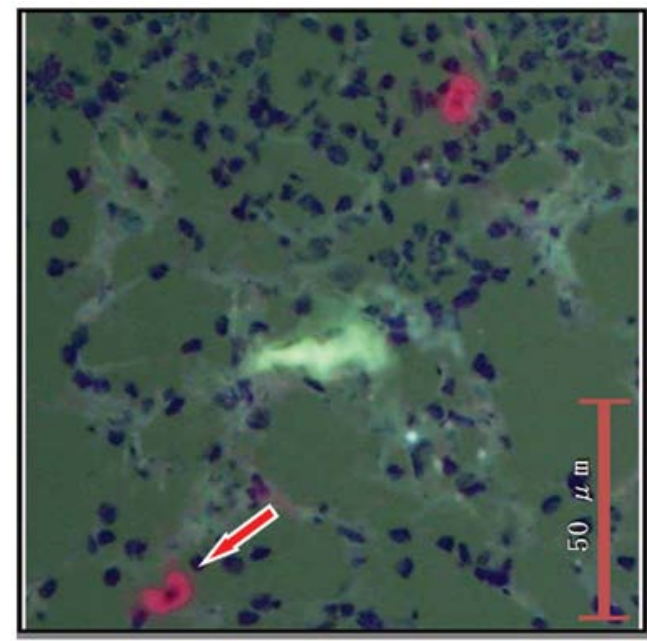

Figure 5. Images of plasma cells in the breast cancer cases. (A) Transmission bright-field observation; (B) epifluorescence.

A

\begin{tabular}{|c|c|c|}
\hline Rapid HE stain & The final pathological diagnosis & RDS method \\
\hline $\begin{array}{ccc}\mathrm{N} 1 & \ldots & 21 \text { cases } \\
& & 28 \text { nodes }\end{array}$ & 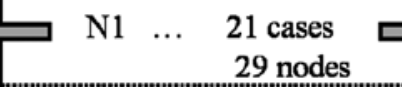 & $\begin{array}{ccc}\mathrm{N} 1 & \ldots & 20 \text { cases } \\
& 27 \text { nodes }\end{array}$ \\
\hline $\begin{array}{c}\text { False negative case : } 0 \% \\
\text { False negative rate of SLN : } 3.45 \%\end{array}$ & & $\begin{array}{c}\text { False negative case : } 4.76 \% \\
\text { False negative rate of SLN : } 6.90 \%\end{array}$ \\
\hline
\end{tabular}

B

\begin{tabular}{|c|c|c|}
\hline Rapid HE stain & The final pathological diagnosis & RDS method \\
\hline \hline $\begin{array}{c}\mathrm{N} 1 \text { (mi) } \quad \ldots 4 \text { cases } \\
4 \text { nodes }\end{array}$ & N1(mi) $\ldots 6$ cases & \multicolumn{2}{c|}{$\begin{array}{c}\text { N1(mi) } \ldots 5 \text { cases } \\
5 \text { nodes }\end{array}$} & $\begin{array}{c}\text { nodes } \\
\text { False negative case }: 33.3 \% \\
\text { False negative rate of SLN }: 33.3 \%\end{array}$ & & $\begin{array}{c}\text { False negative case }: 16.7 \% \\
\text { False negative rate of SLN }: 16.7 \%\end{array}$ \\
\hline
\end{tabular}

C

\begin{tabular}{|c|c|c|}
\hline Rapid HE stain & The final pathological diagnosis & RDS method \\
\hline $\begin{array}{ccc}\mathrm{NO}(\mathrm{i}+) & \ldots & 1 \text { case } \\
& 1 \text { node }\end{array}$ & $\begin{array}{rr}\mathrm{NO}(\mathrm{i}+) & \ldots 5 \text { cases } \\
& 7 \text { node }\end{array}$ & $\begin{array}{r}\text { No(i+) } \ldots 5 \text { cases } \\
6 \text { node }\end{array}$ \\
\hline $\begin{array}{c}\text { False negative case : } \mathbf{8 0 . 0 \%} \\
\text { False negative rate of SLN : } 85.7 \%\end{array}$ & & $\begin{array}{c}\text { False negative case : } 0 \% \\
\text { False negative rate of SLN : } 14.3 \%\end{array}$ \\
\hline
\end{tabular}

Figure 6. Comparison of the false-negative rates between rapid hematoxylin and eosin (HE) staining and the rapid double staining (RDS) methods. (A) Samples from 21 patients diagnosed with N1 by final pathological examination. (B) Samples from 6 patients diagnosed with N1(mi) by final pathological examination. (C) Samples from 5 patients diagnosed with NO(i+) by final pathological examination.

appear orange under epifluorescence microscopy, as do cancer cells. However, H\&E staining facilitates determination of the absence of cancer cells when viewed under bright-field observation.

Diagnosis of metastasis by examining SN in breast cancer. Of the 100 patients, $21(21.0 \%)$ were diagnosed as $\mathrm{pN} 1$ in the final pathological examination. Of the 372 lymph nodes, metastasis was identified in $29(7.8 \%)$. While all 21 cases were diagnosed as having metastatic disease by rapid H\&E staining, 20 cases were diagnosed as having metastatic disease by RDS. Thus, the false-negative rate was $0 \%$ for rapid H\&E staining and $4.8 \%$ for RDS. The reason for the false-negative case by RDS was lack of a metastatic focus in the RDS frozen section; the metastatic focus was likely lost from the section in the slicing step (Fig. 6A).

Of the 100 patients, $6(6.0 \%)$ were diagnosed as $\mathrm{pN} 1(\mathrm{mi})$ in the final pathological examination. Of the $372 \mathrm{lymph}$ nodes, metastasis was identified in $6(1.61 \%)$. We were able to detect metastatic foci in 4 of these 6 cases with rapid H\&E 
staining; metastatic foci were detected in 5 of the cases with RDS. Thus, the false-negative rate was $33.3 \%$ for rapid $H \& E$ staining and $16.7 \%$ for RDS. With regard to the one case in which metastasis was not detected by RDS, the specimen was ultimately determined to be from a case with ITCs rather than micrometastasis (Fig. 6B).

Of the 100 patients, $5(5.0 \%)$ were diagnosed as pN0(i+) in the final pathological examination. Of the 372 lymph nodes, 7 $(1.88 \%)$ had ITCs. While rapid H\&E staining detected ITCs in only 1 of the 5 cases, RDS detected ITCs in all 5 cases. Thus, the false-negative rate was $80.0 \%$ for rapid $\mathrm{H} \& \mathrm{E}$ staining and $0 \%$ for RDS. RDS failed to detect ITCs in 1 lymph node, probably due to a lack of cancer cells in the frozen section (Fig. 6C).

\section{Discussion}

In the present study, we demonstrated that RDS was superior to conventional rapid H\&E staining for the diagnosis of ITCs. This method, therefore, represents a promising improvement in the accuracy of frozen section diagnosis of sentinel-node biopsy.

Needless to say, in order to achieve more accurate diagnoses of metastases, it will be necessary to improve the intraoperative diagnostic effectiveness of the procedure $(13,14)$.

In the present study, the accuracy of diagnosis of intraoperative rapid $H \& E$ was very high $(91 \%)$. To improve the accuracy of intraoperative diagnosis of lymph node metastases, we cut lymph nodes into $2-\mathrm{mm}$ slices and prepared multiple slices (6). However, for breast cancer, the accuracy of intraoperative frozen section diagnosis of metastatic lymph node lesions (metastatic lesions $>0.2 \mathrm{~mm}$ ) varies among studies (1). For example, Ali et al (1) reported the accuracy to be $76 \%$, while Tanis et al (15) found it to be $74 \%$. To increase the accuracy of detection of lymph node metastases, multiple intraoperative immunohistochemical evaluation methods using anti-cytokeratin antibodies have been developed $(16,17)$. Many of these staining procedures, however, are complicated, time-consuming and therefore impractical.

In order to observe the immunostaining results more clearly, we used a fluorescence immunostaining method (9). Traditionally, fluorescence immunostaining has been available only as a two-step procedure in which a luminescence reagent is added after antibody molecules are bound to cells. Such a technique has multiple drawbacks: the procedure is timeconsuming and the period of light emission is short (8).

To overcome these drawbacks, we developed RDS, which has the following characteristics: a convenient one-step procedure for immunostaining, very clear staining results, rapid $\mathrm{H} \& \mathrm{E}$ and immunofluorescence staining of the same slice of specimen, easy staining procedures, no requirement for special equipment except for a fluorescence microscope and low cost.

Using the RDS method, the limitations of the fluorescence immunostaining method were overcome by using nanocrystal beads, which allow for clearer visualization and a shorter procedure time. We used QDs as nanocrystal beads. The energy acceptor modules consisted of $\mathrm{CdSe} / \mathrm{ZnS}$ semiconductor nanocrystals coated with a polymer shell containing 5-7 biotin molecules per dot on their surfaces (18). The beads also have stable, bright optical characteristics; furthermore, through a maleimide-thiol binding reaction, they can be conjugated with any antibody (18). Various QDs are available, each with a different emission spectrum (19). Herein, we used QD655, which produces maximal emission at $655 \mathrm{~nm}$. The color of QD655 fluorescence is red, clearly contrasting with normal lymphocytes, which appear green upon eosin staining. Based on the procedure reported in a study by Ishii et al (3), we conjugated these QDs to anti-cytokeratin 8/18 antibodies (SC-8020).

As frozen-section diagnosis at our hospital is highly effective and accurate overall, the advantage of RDS in this study was limited to the diagnosis of ITCs (4). Nevertheless, we believe that RDS has sufficient value for clinical use. In many institutions, if the diagnosis of ITCs can be established by intraoperative rapid diagnostic methods applied to lymph nodes, axillary lymph node dissection is not routinely performed (1). Galimberit et al (20) reported, however, that $15-19 \%$ of nonsentinel nodes were found to be metastatic in cases where ITCs were present in SNs. In a study by de Boer et al (4), the presence or absence of micrometastases and ITCs was also shown to be associated with survival in patients who did not receive postoperative adjuvant chemotherapy. Additionally, a case in which RDS failed to find micrometastasis was diagnosed as having ITCs. We speculate that this false-negative case was attributable to issues related to preparation of the sections; a micrometastasis is very small and a section does not always represent the maximal cutting size. Therefore, the diagnosis should be made intraoperatively in any case, although whether lymph node dissection is needed for patients with ITCs is a separate issue that needs to be addressed $(21,22)$.

For rapid diagnosis of lymph node metastasis, one-step nucleic acid amplification (OSNA) assay was recently developed (23). With this method, the expression level of cytokeratin 19 (CK19) mRNA is assessed with specific primers, and amplification and detection of CK19 mRNA can be achieved in $230 \mathrm{~min}$. Tamaki et al (24) reported the sensitivity and specificity of the OSNA assay for detection of metastases to be 95 and $97.1 \%$, respectively.

Some metastatic lymph nodes of breast cancer patients, however, are CK19-negative. The OSNA assay may provide false-negative results in such cases (24). Parikh et al (25) reported lack of CK19 expression in $20.5 \%$ of 158 breast carcinomas in a tissue microarray. Moreover, they found a statistically significant association between lack of CK19 expression and the triple-negative (TN) phenotype (30\% of TN breast cancers were CK19-negative). Since the RDS method also detects the expression level of cytokeratin 8/18 (CK8/18), CK8/18-negative metastatic lymph nodes would display no fluorescence signals. While no such cases were observed in this study, our RDS method would avoid such false-negative results by switching filters for bright-field observation, allowing morphological detection of cancerous structures by $H \& E$ staining of the same section.

Moreover, in the OSNA assay, sample lymph nodes must be homogenized in lysis buffer. Thus, generally, when performing a rapid intraoperative diagnosis, half of the lymph node sample is used for the OSNA assay and the other half for imprint cytology (24). For the samples used for OSNA, morphological information regarding lymph node metastases is completely lost during the lysis step and consequently cannot be used later 
to confirm the diagnosis. Unlike the OSNA assay, the RDS method allows morphological examination for the detection of metastases, because the same frozen sections are used for fluorescence immunostaining and H\&E staining.

RDS would also be useful for SN biopsy in gastrointestinal cancer cases. Since these patients cannot be re-operated, the presence or absence of SN micrometastases must be diagnosed intraoperatively.

As long as tumor cells are present in the sections, the RDS method facilitates the diagnosis of ITCs even when involvement is minimal. Our RDS method is clinically useful for examination of the SN biopsy of breast cancers and can contribute to the intraoperative diagnosis of lymph node metastases and standardization of cancer treatments.

\section{Acknowledgements}

The authors are grateful to Dr Koichi Miwa of Kanazawa University for making the present study possible.

\section{References}

1. Ali R, Hanly AM, Naughton P, et al: Intraoperative frozen section assessment of sentinel lymph nodes in the operative management of women with symptomatic breast cancer. World J Surg Oncol 6: 69, 2008.

2. Giobuin SM, Kavanagh DO, Myers E, et al: The significance of immunohistochemistry positivity in sentinel nodes which are negative on haematoxylin and eosin in breast cancer. Eur J Surg Oncol 35: 1257-1260, 2009.

3. Gobardhan PD, Elias SG, Madsen EV, et al: Prognostic value of micrometastases in sentinel lymph nodes of patients with breast carcinoma: a cohort study. Ann Oncol 20: 41-48, 2009.

4. de Boer M, van Deurzen CH, van Dijck JA, et al: Micrometastases or isolated tumor cells and the outcome of breast cancer. N Engl J Med 361: 653-663, 2009.

5. Mikhitarian K, Martin RH, Mitas M, et al: Molecular analysis improves sensitivity of breast sentinel lymph node biopsy: results of a multi-institutional prospective cohort study. Surgery 138 : 474-481, 2005

6. Fritzsche FR, Reineke T, Morawietz L, et al: Pathological processing techniques and final diagnosis of breast cancer sentinel lymph nodes. Ann Surg Oncol 17: 2892-2898, 2010.

7. Tsujimoto M, Nakabayashi K, Yoshidome K, et al: One-step nucleic acid amplification for intraoperative detection of lymph node metastasis in breast cancer patients. Clin Cancer Res 13 4807-4816, 2007.

8. Ishii K, Kinami S, Funaki K, et al: Detection of sentinel and non-sentinel lymph node micrometastases by complete serial sectioning and immunohistochemical analysis for gastric cancer. J Exp Clin Cancer Res 27: 7, 2008.

9. Chattopadhyay PK, Price DA, Harper TF, et al: Quantum dot semiconductor nanocrystals for immunophenotyping by polychromatic flow cytometry. Nat Med 12: 972-977, 2006.
10. Chung I, Witkoskie JB, Cao J and Bawendi MG: Description of the fluorescence intensity time trace of collections of CdSe nanocrystal quantum dots based on single quantum dot fluorescence blinking statistics. Phys Rev E Stat Nonlin Soft Matter Phys 73: 011106, 2006.

11. Schmidt H, Brown EB, Schwaller B and Eilers J: Diffusional mobility of parvalbumin in spiny dendrites of cerebellar Purkinje neurons quantified by fluorescence recovery after photobleaching. Biophys J 84: 2599-2608, 2003.

12. Noguchi M, Inokuchi M and Zen Y: Complement of peritumoral and subareolar injection in breast cancer sentinel lymph node biopsy. J Surg Oncol 100: 100-105, 2009.

13. Turner RR, Ollila DW, Stern S and Giuliano AE: Optimal histopathologic examination of the sentinel lymph node for breast carcinoma staging. Am J Surg Pathol 23: 263-267, 1999.

14. Viale G, Dell'Orto P, Biasi MO, et al: Comparative evaluation of an extensive histopathologic examination and a real-time reversetranscription-polymerase chain reaction assay for mammaglobin and cytokeratin 19 on axillary sentinel lymph nodes of breast carcinoma patients. Ann Surg 247: 136-142, 2008.

15. Tanis PJ, Boom RP, Koops HS, et al: Frozen section investigation of the sentinel node in malignant melanoma and breast cancer. Ann Surg Oncol 8: 222-226, 2001.

16. Hughes SJ, Xi L, Raja S, et al: A rapid, fully automated, molecular-based assay accurately analyzes sentinel lymph nodes for the presence of metastatic breast cancer. Ann Surg 243: 389-398, 2006.

17. Carmon M, Olsha O, Rivkin L, Spira RM and Golomb E: Intraoperative palpation for clinically suspicious axillary sentinel lymph nodes reduces the false-negative rate of sentinel lymph node biopsy in breast cancer. Breast J 12: 199-201, 2006.

18. Lifshitz E, Brumer M, Kigel A, et al: Air-stable PbSe/PbS and $\mathrm{PbSe} / \mathrm{PbSexS1-x}$ core-shell nanocrystal quantum dots and their applications. J Phys Chem B 110: 25356-25365, 2006.

19. Charbonnière LJ, Hildebrandt $\mathrm{N}$, Ziessel RF and Löhmannsröben HG: Lanthanides to quantum dots resonance energy transfer in time-resolved fluoro-immunoassays and luminescence microscopy. J Am Chem Soc 128: 12800-12809, 2006.

20. Galimberti V: Axillary sentinel lymph node: how low can you go? Breast 18 (Suppl 1): S12, 2009.

21. Nissan A, Jager D, Roystacher M, et al: Multimarker RT-PCR assay for the detection of minimal residual disease in sentinel lymph nodes of breast cancer patients. Br J Cancer 94: 681-685, 2006.

22. Bostick PJ, Huynh KT, Sarantou T, et al: Detection of metastases in sentinel lymph nodes of breast cancer patients by multiplemarker RT-PCR. Int J Cancer 79: 645-651, 1998.

23. Schem C, Maass N, Bauerschlag DO, et al: One-step nucleic acid amplification - a molecular method for the detection of lymph node metastases in breast cancer patients; results of the German study group. Virchows Arch 454: 203-210, 2009.

24. Tamaki Y, Akiyama F, Iwase T, et al: Molecular detection of lymph node metastases in breast cancer patients: results of a multicenter trial using the one-step nucleic acid amplification assay. Clin Cancer Res 15: 2879-2884, 2009.

25. Parikh RR, Yang Q, Higgins SA and Haffty BG: Outcomes in young women with breast cancer of triple-negative phenotype: the prognostic significance of CK19 expression. Int J Radiat Oncol Biol Phys 70: 35-42, 2008. 